\title{
A Real-Time Hand Interaction System for Image Sensor Based Interface
}

\author{
SeIn Lee ${ }^{1}$, Jonghoon Seo ${ }^{1}$, Soon-bum Lim ${ }^{2}$, Yoon-Chul Choy ${ }^{1}$, \\ and TackDon $\operatorname{Han}^{1}$ \\ ${ }^{1}$ Dept. Of Computer Science, Yonsei University, \\ 134, Sinchon-dong, Seodaemun-gu \\ \{sipkjy, jonghoon. seo, ycchoy, hantack\} @yonsei.ac.kr \\ 2 Dept. Of Multimedia Science, Sookmyung University, \\ 52 Hyochangwon-gil, Yongsan-gu, \\ Seoul, Korea \\ sblimasookmyung.ac.kr
}

\begin{abstract}
Diverse sensors are available in ubiquitous computing of which resources is inherent in environment. Among them, image sensor acquires necessary data using camera without any extra devices, which is a different aspect from other sensors. It can provide additional services and/or applications by using a location of code and ID in real time image. Focusing on this, Intuitive interface operating method in ubiquitous computing environment that has plenty of image codes is suggested. GUI using image sensor was designed, which works real-time interactive operation between user and the GUI without any additional button or device. This interface method recognizes user's hand images in real-time by learning them at a starting point. The method sets interaction point, and operates the GUI through hand gestures defined previously. We expect this study can be adopted to augmented reality area and real time interface using user's hand.
\end{abstract}

Keywords: HCI, Hand interface, Hand interaction, Augmented Reality.

\section{Introduction}

In 2008, Microsoft's Bill Gates emphasized the importance of convenient interfaces using the phrase "second digital decade."[1] However, there are many inconveniences with the interface. Input inconvenient and lacking in intuition, thus necessitating additional learning. In contrast, the second digital decade interfaces need to be more intuitive, user-friendly, and convenient to use. We suggest an interaction system that satisfies the needs for future ubiquitous computing environment. Various information and applications can be used in ubiquitous computing environment. The ubiquitous computing environment is assumed for the study, in which digital information used has a $2 \mathrm{~d}$ image code form. We are considering image sensor based real time and real environment service or application under the environment. This is a kind of augmented reality (AR) approach. In this paper, as a method to interact with GUI in AR environment, we present a system that directly uses user's hand. 


\section{AR Interaction Analysis}

Depending on the interaction modes used, augmented reality interfaces can be classified into tangible user interface (TUI) based method, control device based method, or hand based methods. There are diverse control devices available, with the keyboard and the mouse being the most common. Examined here is an example of AR utilization in a hand-held device that employs the touch screen and tablet pen based interface, which is lately gaining increased application.

The method most easily and often used for AR interface is the TUI based interface. In general, the TUI utilization method employs as the physical tool for interaction, a 2D image marker based on an image processing marker recognition method. When this is done, the marker is either attached to a stick or made so that it can be hand-held in order to prevent the marker's image part from being occluded $[2,3,4,5,6]$. Or in certain situations, a special TUI [7] is created. When an image marker is used, since the decoding algorithm of the marker is already established, construction of additional physical user interface is convenient and easy. However, if the marker is occluded during interaction, the feedback from the marker is lost, and thus there is a constraint of not having any part of the marker occluded during operation. Furthermore, since the decoding of one ID is done by one marker, in order to carry out complex manipulations, the number of TUI has to be increased by a corresponding amount. This creates a shortcoming in that the interaction becomes uncomfortable and manipulation unnatural. The non-vision based, specially produced TUI can resolve this to some extent, but it cannot be easily built since from production to interaction it requires special devices.

The touch screen based method $[8,9,10,11]$, with a $2 \mathrm{D}$ interface familiar to general users, makes easy manipulation possible. But if it is used in mixture with the AR environment, confusion occurs in interaction. While the elements for UI augmentation utilize real environment resources, the user's manipulations take place in the device's 2D screen. Therefore, it only serves the role of receiving information inherent in the environment without any interaction with a physical medium, and the virtual object, rather than being directly controlled, is indirectly manipulated.

There are various forms of interaction using the hand within the AR environment. There is the method of using a special device like a glove [12], and as in [13], there is the occlusion based method using occlusion of the marker in an image. The manipulation method using the shadow of a hand on a touch creen was also employed, as shown in [11]. These methods employ the hand and at the same time require other resources in addition to the hand. In contrast, the most natural and simple method is the vision based method which directly uses just the hand. The vision based method is employed in [14]. This method, however, only utilizes the single, most prominently recognized finger and thus additionally requires a control panel for manipulation, which tends to diminish intuitiveness.

Focusing on intuitiveness and naturalness, we created a gesture interaction system through a vision based recognition method. This uses natural and familiar gestures employing just the hand without a TUI or supplementary devices. A detailed explanation of our method will be given in Chapter 3 . 


\section{Personal Hand Interface System}

We are considering developing an application that augments service or GUI using 2D image sensors afterwards. As preparatory research for the proposal, this paper researched technology that recognizes hands using neural network and uses them interactively.

\subsection{System Processing}

The system operates as shown in Figure 1. When the program is first run, it goes through the learning process of extracting user's hand information. Through it a neural network is composed and the process moves to hand recognition stage. In the hand recognition stage, the individual user's hand is recognized and then, in the gesture recognition stage, gestures are discerned based on hand shapes. Afterwards, interaction is carried out according to changes in the gestures.
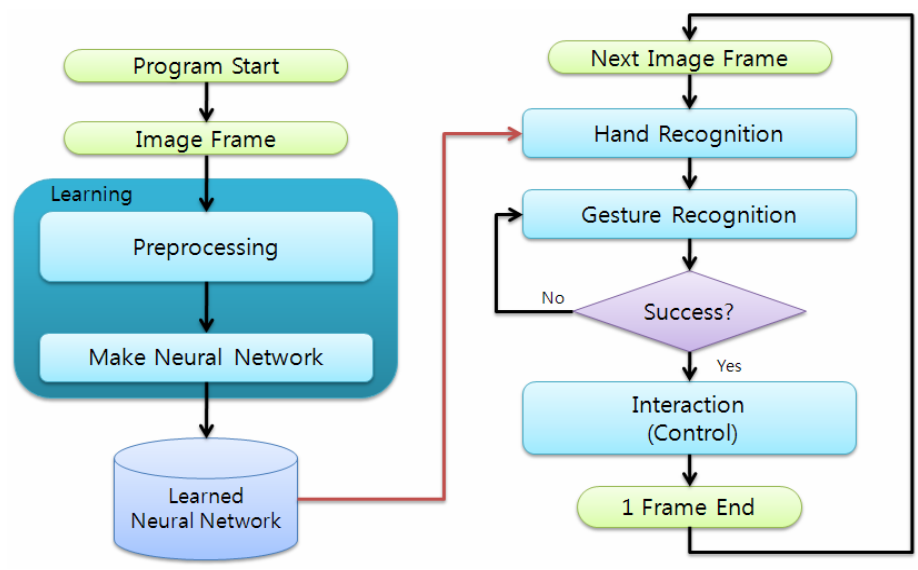

Fig. 1. The processing architecture

\subsection{Learning}

In the learning stage, the features of a user's hand are identified and a neural network is established. To find the features, when the user places a hand on the circular mark at the center of the picture as shown in Figure 2(a), the R, G, B, H, S, V, Y, Cb, Cr color tones of the circular mark area are extracted. The RGB model is the basic system model, the HSV model is good for detecting changes in color tones, and because the $\mathrm{YCbCr}$ model is good for accurately sorting out luminance and chrominance, it is mainly used for skin color modeling.[9]

The characteristics thus extracted are used to learn a neural network. Figure 2(b) shows the structure of a neural network realized in the system, and it is composed of an input layer which inputs nine types of color value averages from user's hand, a two column hidden layer made up of 30 neurons, and one output neuron. The system was taught to make the output neuron converge to zero. 


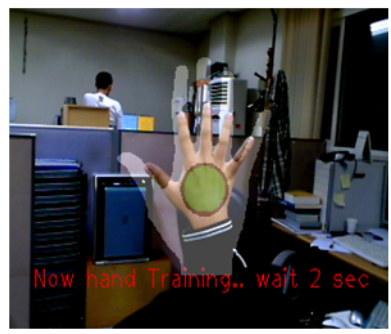

(a) User hand learning

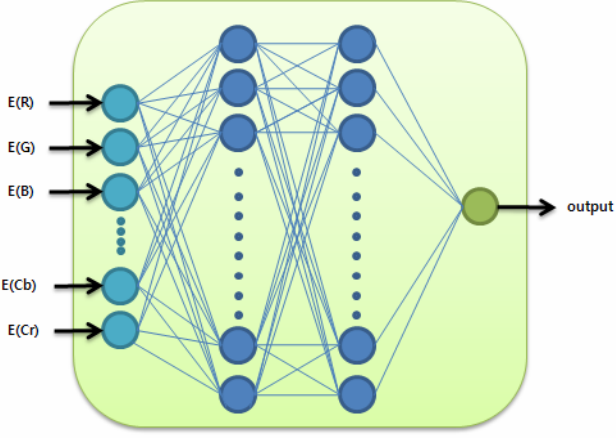

(b) Neural network

Fig. 2. Learning stage via (a) user's hand learning image (b) neural network model

\subsection{Hand Recognition}

Once learning is completed, the recognition stage is carried out. From the image, candidate areas deemed to be the skin color area are identified through the average and standard deviation of $\mathrm{Y}, \mathrm{Cb}, \mathrm{Cr}, \mathrm{H}$ elements. Based on the silhouette pixel number $\mathrm{k}$ from the user hand data extracted during the learning stage, among the candidate areas, objects that are too small or too big are judged to be noise and eliminated.

From all the objects left on the screen, the color values that match those from the learning stage are obtained and their average is calculated. Then the neural network produced from the learning stage is recalled, and the resulting value of each object is tested. Because the system was taught to make the resulting value of the output neuron converge to zero in the learning stage, from among the objects that have been tested, the one with the resulting value approaching closest to zero is judged to be user's hand.

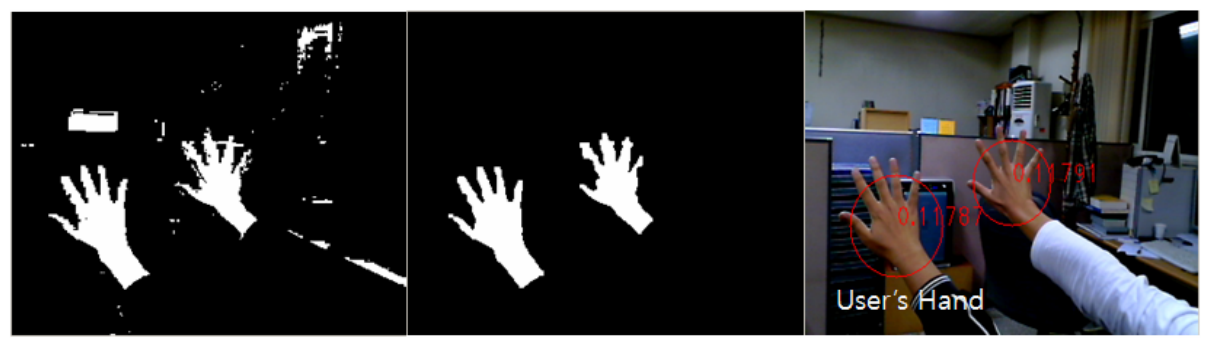

Fig. 3. Hand recognition stage \{binary, noise reduced, neural network value attached $\}$ images

\subsection{Gesture Recognition}

The hand image area and centroid coordinates were obtained, and the radius was established according to the area ratio. Then the hand is explored along a circle to confirm the number of fingers and tip points in the hand are selected. While processing images 
in real-time, there can be a problem of abnormal value calculations with finger count, center of gravity, or radius momentarily occurring due to unexpected changes in the images. In order to prevent this, another state is added into the middle of the changing states, and this prevents error from occurring. When the number of fingers changes, if the changed state is maintained with a delay of three frames, then the state is registered as changed.

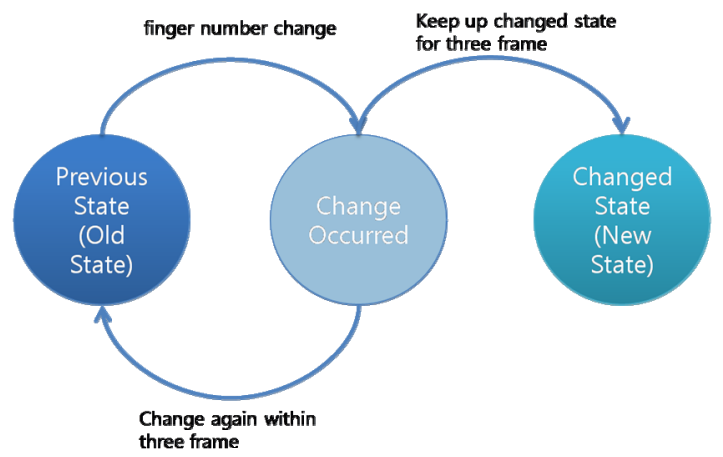

Fig. 4. Gesture state change graph

\subsection{Interaction Design}

There are a total of six gesture types that users can use. To enable the user to easily learn the operating method, in considering the gestures we sought natural motions or familiar motions of a common interface such as "Grasp" and "Click" of Table 1. Table 1 presents the gesture descriptions.

\begin{tabular}{|l|l|l|l|l|l|l|} 
& 1 Finger & Click & 2 Finger & Grasp & F Finger & Fist \\
\hline Interaction & - Object select & $\begin{array}{l}\text { - Confirm } \\
\text { selection }\end{array}$ & $\begin{array}{l}\text { - Object select } \\
\text { - Drag release }\end{array}$ & $\begin{array}{l}\text { - Dragging } \\
\text { - Confirm } \\
\text { selection }\end{array}$ & $\begin{array}{l}\text { - Predefined } \\
\text { event }\end{array}$ & $\begin{array}{l}\text { Predefined } \\
\text { event }\end{array}$ \\
\hline Example & & & &
\end{tabular}

Fig. 5. Design of gestures

\section{User Study}

To evaluate the constructed system, the test participants were required to fill out a survey after using the system. Seven users, ages 25 to 30, participated in the test. In order to aid users to make comparative evaluations, the testing was done with the additional inclusion of the TUI based and touchscreen methods. The tasks required of the participants were scaling, rotation and translation, the basic manipulations for $3 \mathrm{D}$ 
Table 1. The detailed description of the three interactions used in the experiments

\begin{tabular}{|c|c|c|c|}
\hline & TUI based & Hand Gesture based & Touchscreen based \\
\hline Tools & Cube type TUI & User's hand & $\begin{array}{l}\text { Tablet pen } \\
\text { Touch screen }\end{array}$ \\
\hline $\begin{array}{l}\text { Interaction } \\
\text { method }\end{array}$ & $\begin{array}{l}\text { Dialing interaction(TUI) } \\
\text { Image marker changing }\end{array}$ & Hand Gesture & $\begin{array}{l}\text { Touch by tablet pen } \\
\text { and press Right button }\end{array}$ \\
\hline Description & $\begin{array}{l}\text { Rotate L/R: turn the TUI } \\
\text { Scale P/M: turn the TUI } \\
\text { Translate: move the TUI } \\
\text { Change manipulation: marker } \\
\text { switch }\end{array}$ & $\begin{array}{l}\text { Rotate L/R: grasping gesture } \\
\text { or click gesture and move } \\
\text { Scale P/M: grasping gesture } \\
\text { or click gesture and move } \\
\text { Translate: grasp gesture and move } \\
\text { or click gesture and move } \\
\text { Change manipulation: five finger } \\
\text { gesture }\end{array}$ & $\begin{array}{l}\text { Rotate L/R: touch and move } \\
\text { Scale P/M: touch and move } \\
\text { Translate: touch and move } \\
\text { Change manipulation: right } \\
\text { button }\end{array}$ \\
\hline
\end{tabular}

objects. The details are presented in table 1. After verbally explaining to the participants how to do the manipulations, they were requested to do the test right away.

\subsection{Usability Survey and Result}

The participants were each requested do the same task employing 3 methods and then fill out the survey. The survey included six items of evaluation: easy to learn, intuitiveness, naturalness, immersion, accuracy of control, and preference. We surveyed the user response by employing seven interval scale items-1 for "very low," 2 for "low," 3 for "little low," 4 for "medium," 5 for "little high," 6 for "high," and 7 for "very high." The results are shown in Figure 6.

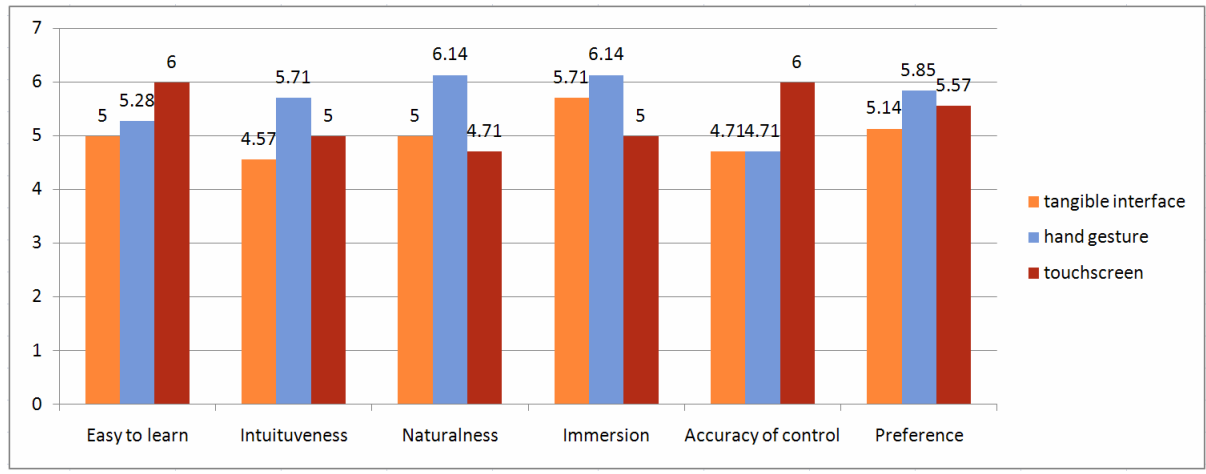

Fig. 6. Result of the survey(avg) 


\subsection{Discussion}

First, regarding the question of manipulation accuracy, the users gave the highest evaluation to the touch screen method. The respondents considered manipulation to be relatively difficult in the remaining two methods. It appears that this was due to the difference in 3D and 2D interfaces as well as the existing experience of the users. Since all the participants had experience of using a touch screen, it was the method most familiar to them. Hence it required less supplementary instructions for learning how to use it. The other two methods, based on observing the users, showed that although they listened to the instructions, their manipulations were awkward at the start and gradually improved as time passed.

Next, the participants were asked how intuitive and natural each method was, and they gave the highest evaluation to the hand gesture method. They found there was no feeling of incongruity with this method since part of the body was directly used and the gestures were easy. The TUI method was not difficult, but they responded that using only a marker made it difficult to intuitively infer the method of use. The touch screen was found to have relatively less natural feeling.

As for immersion, the participants gave the lowest evaluation to the touch screen and positive marks to the other two methods. This is due to the difference in interaction space: for the TUI method and the hand gesture method, manipulation takes place directly in the physical space photographed as an image and the feedback occurs simultaneously; whereas, for the touch screen, the manipulation takes place in a $2 \mathrm{D}$ screen, but the interaction with the virtual object occurs in a real-time image (physical space).

\section{Conclusion and Future Works}

In order to enable users to natural control an enhanced GUI this paper researched a technology that recognizes hands and, with it, analyze gestures and provide interaction. Finally, evaluation criteria were presented and comparative evaluation using a usability test was done on the system we created and the other two methods. The results of the evaluation showed that the users found our method as the most natural and intuitive interface. It was also confirmed that there is a need to improve the accuracy of system manipulation and that users prefer simpler gesture based method than is currently available. This technique is expected to be applied to an augmented reality system in the future and be utilized as its interface for a more natural interaction.

\section{Acknowledgement}

This research was supported by the Ministry of Education \& Human Resources Development BK21 and the Basic Research Program of Korea Science and Engineering Foundation (R01-2005-000-10898-0 / R01-2008-000-20849-0 ). 


\section{References}

1. Gates, B.: World to enter 'second digital decade' - Times Online, http://technology.timesonline.co.uk/tol/news/tech_and_web/ article3144255.ece

2. Lee, G.A., Nelles, C., Billinghurst, M., Kim, G.J.: Immersive authoring of Tangible Augmented Reality applications. In: International Symposium on Mixed and Augmented Reality, pp. 172-181 (2005)

3. Kim, L., Cho, H., Park, S.H., Han, M.: A Tangible User Interface with Multimodal Feedback. In: Schmorrow, D.D., Reeves, L.M. (eds.) HCII 2007. LNCS, vol. 4565, pp. 94-103. Springer, Heidelberg (2007)

4. Zhou, Z., Cheok, A.D., Chan, T., Pan, J.H., Li, Y.: Interactive Entertainment Systems Using Tangible Cubes. In: Australian Workshop on Interactive Entertainment (IE 2004), pp. 19-22 (2004)

5. Billinghurst, M., Kato, H., Poupyrev, I.: Tangible Augmented Reality. International Conference on Artificial Reality and Telexistence (ICAT) Tutorial (2004)

6. Moore, A., Regenbrecht, H.: The Tangible Augmented Street Map. In: International Conference on Artificial Reality and Teleexistence (ICAT), pp. 249-250 (2005)

7. Sinclair, P., Martinez, K.: Adapting Information Through Tangible Augmented Reality Interfaces. In: UbiComp 2004 (2004)

8. Mooser, J., Wang, L., You, S., Neumann, U.: An Augmented Reality Interface for Mobile Information Retrieval. In: International Conference on Multimedia and Expo (ICME), pp. 2226-2229 (2007)

9. Wagner, D., Schmalstieg, D.: First Steps Towards Handheld Augmented Reality. In: 7th International Conference on Wearable Computers (ISWC 2003), pp. 127-135 (2003)

10. Wilson, A.D.: PlayAnywhere: a compact interactive tabletop projection-vision system. In: UIST 2005, pp. 83-92 (2005)

11. Wagner, D., Pintaric, T., Ledermann, F., Schmalstieg, D.: Towards massively multi-user augmented reality on handheld devices. In: Gellersen, H.-W., Want, R., Schmidt, A. (eds.) Pervasive 2005. LNCS, vol. 3468, pp. 208-219. Springer, Heidelberg (2005)

12. Dorfmüller-Ulhaas, K., Schmalstieg, D.: Finger tracking for interaction in augmented environments. In: International Symposium on Augmented Reality, pp. 55-64 (2001)

13. Lee, G.A., Billinghurst, M., Kim, G.J.: Occlusion based Interaction Methods for Tangible Augmented Reality Environments. In: International conference on Virtual Reality continuum and its applications in industry, pp. 419-426 (2004)

14. McDonald, C., Roth, G.: Hand Based Interaction in Augmented Reality. In: IEEE International Workshop on Haptic Audio Visual Environments and their Applications (HAVE 2002), pp. 55-59 (2002) 